\title{
Hypersensitivity reaction to dapsone: report from Malaysia
}

\author{
KADER NAINA MOHAMED \\ Department of Dermatology, General Hospital, Johor Bahru, \\ Malaysia
}

Accepted for publication 30 May 1984

\begin{abstract}
Summary Three patients who developed hypersensitivity reaction at varying intervals after the initiation of dapsone therapy are described. Two of them had leprosy whereas the other had dermatitis herpetiformis. Their clinical manifestations were not uniform. Although fatal complications have been reported, the unique role played by dapsone in the treatment of leprosy and the importance of recognizing this reaction are discussed.
\end{abstract}

\section{Introduction}

Dapsone is a very familiar sulphonamide to those who work in the field of leprosy. It is also used by the dermatologist for the treatment of chronic bullous disorders-dermatitis herpetiformis, ${ }^{1}$ Hailey-Hailey disease, ${ }^{2}$ bullous pemphigoid, and various other conditions such as subcorneal pustular dermatosis, erythema elevatum diutinum, acne conglobata and leucocy toclastic vasculitis. ${ }^{3}$ In developing countries like Malaysia where dermatologists are actively involved in the management of leprosy patients, the beneficial effect of this drug for both skin disorders and leprosy can be easily observed and appreciated.

Dapsone is a competitive inhibitor of para-aminobenzoic acid, and thereby interferes with the synthesis of folic acid. Nevertheless, how it exerts its bacteriostatic effect in leprosy is not well established. The mechanism of action in dermatitis herpetiformis is believed to be that of inhibition of the alternate pathway in the complement system and that of polymorpho-leucocyte cytotoxicity. ${ }^{4}$ Dapsone is well absorbed when administered orally and about $50-80 \%$ is plasma-bound. ${ }^{5}$ It undergoes enterohepatic circulation and is subjected to acetylation and renal excretion as glucuronyl conjugate. Its half-life is around $21 \mathrm{~h}$.

The side-effects of dapsone are methaemoglobinaemia, haemolytic anaemia, megaloblastic anaemia which are dose-related, agranulocytosis, peripheral neuropathy, nephrotic syndrome ${ }^{6}$ and hypo-albuminaemia. ${ }^{7}$ Hypersensitivity 
reactions, rarely seen nowadays were observed as early as 1950 and named as dapsone or DAPT Syndrome ${ }^{8}$ which was characterized by fever, generalized rash, lymphadenopathy, leucocytosis involving usually the monocytes and jaundice which occurred within the first 6 weeks of dapsone treatment. Recently, the author encountered dapsone hypersensitivity in 2 patients with leprosy and 1 with dermatitis herpetiformis.

\section{Case reports}

Case 1. A 17-year-old Malay girl presented with erythematous, annular rash on the right palm and dorsum of left leg with impaired sensation and thickening of the corresponding ulnar and lateral popliteal nerves with slight tenderness. The bacterial index (BI) was 2.6 and morphological index (MI) was $3 \cdot 1 \%$. Skin biopsy for histopathology and mouse foot-pad inoculation for detection of primary dapsone resistance was done. Other relevant investigations such as full blood picture, liver function tests (LFT), blood urea and Glucose-6-Phosphate-dehydrogenase (G6PD) were within normal limits. She was started on rifampicin 600 $\mathrm{mg}$ and dapsone $100 \mathrm{mg}$ daily. Histopathology confirmed the clinical diagnosis of borderline lepromatous (BL) leprosy. On the third week of the antileprosy treatment when the MI had become 0 and the BI was $2 \cdot 3$, the patient suddenly developed fever, generalized rash and swelling and tenderness of cervical group of lymph nodes and bluish discolouration of lips and nails. The temperature was $40^{\circ} \mathrm{C}$.

The first symptom to appear was the generalized erythematous rash whose distribution was mainly on the face, trunk and limbs with minimal involvement of the palms and soles. The eyes, mouth and the genitalia were not affected. The rash was predominantly macular with few papules scattered over the abdomen. The nerves were slightly tender, the initial skin lesions on the palm and foot did not become very erythematous or shiny; no hepatic tenderness or jaundice, ESR was $45 \mathrm{~mm}$ and LFT was within normal limits. Peripheral blood film showed haemolytic anaemia. There was no tender nodule and eyes were clear. Paul-Bunnell test and repeat skin biopsy were not done. The clinical picture was not that of reversal or type I lepra reaction and the chain of symptoms was more in favour of drug hypersensitivity. Therefore all drugs were stopped and general supportive treatment instituted. Within 2 days the rash faded without leading on to exfoliative dermatitis, the temperature dropped to normal, cervical lymph nodes became less tender and methamoglobinaemia disappeared. One week after she was back to normal, dapsone $25 \mathrm{mg}$ was given, $6 \mathrm{~h}$ later she developed a temperature of $39^{\circ} \mathrm{C}$ with headache and no vomiting. This proved that she manifested dapsone hypersensitivity. She was started on clofazimine, $50 \mathrm{mg}$ daily.

Case 2. An 18-year-old Malaysian Aboriginee girl was seen with an annular patch about $10 \mathrm{~cm}$ in diameter with a central area of hypopigmentation and 
hypoanaesthesia on the right gluteal region. The BI was 0 . Skin biopsy confirmed borderline tuberculoid (BT) leprosy. After performing other relevant tests she was started on dapsone $100 \mathrm{mg}$ daily. The following day after she was given a second dose of dapsone she developed generalized morbilliform rash with loss of appetite and a temperature of $39^{\circ} \mathrm{C}$. On the third day she developed jaundice, cervical lymph node enlargement, but no cynosis. The ESR was $62 \mathrm{~mm}$, serum glutamic-oxaloacetic transaminase was $48 \mathrm{IU}$, bilirubin $5.6 \mathrm{mg} / \mathrm{dl}$. Peripheral blood film was normal. It was not known if she had ever taken dapsone or any other sulphonamide previously. She did not warrant prednisolone and her condition returned to normal within a week on withdrawal of dapsone and on symptomatic treatment. Later she was started on clofazimine $50 \mathrm{mg}$ daily.

Case 3. A 61-year-old Pakistani man was seen with extremely pruritic vesicles, of one month's duration, distributed over the forearms, neck and back of chest and lumbar region. Skin biopsy confirmed the diagnosis of dermatitis herpetiformis $(\mathrm{DH})$. Relevant investigation which included G6PD activity were normal. He was started on dapsone $100 \mathrm{mg}$ daily which was gradually increased to $200 \mathrm{mg}$ daily, according to clinical response. After 4 weeks of dapsone therapy, the patient developed a high fever, $40 \cdot 5^{\circ} \mathrm{C}$, cervical lymph node enlargement and hepatic tenderness, but no jaundice was seen. Vesicles of DH reappeared but generalized macular or papular rash was not observed. The total white cell count was high, 21,500. No evidence of infection was noted. Dapsone was stopped and when clinical and biochemical parameters had become normal, he was given dapsone $50 \mathrm{mg}$. The following day his symptoms recurred. Subsequently he responded well to Sulphapyridine $500 \mathrm{mg}$ tds.

\section{Discussion}

In Malaysia, dapsone was used in 1948 at Sungai Buloh Leprosarium. The author's centre has about 570 leprosy patients in the follow-up, most of them receiving dapsone as maintenance therapy. The 3 patients reported here are the only cases known to have developed dapsone hypersensitivity in this region. A syndrome which included fever, mononucleosis, splenomegaly, hepatitis, and exfoliative dermatitis was first described by Lowe \& Smith in $1949^{9}$ in patients receiving large doses of dapsone. They called it 'glandular fever precipitated by sulphone therapy' as they found a rise in Paul-Bunnell titre. Allday \& Barnes ${ }^{8}$ in 1951 gave it the name 'DADPS syndrome' and were the first to put forward the view that it was a hypersensitivity syndrome. Their dosage of dapsone was 100 $\mathrm{mg} /$ day . slowly increasing to $200 \mathrm{mg} /$ day. These authors stressed that the syndrome invariably developed 5-6 weeks after commencing dapsone therapy and was sometimes fatal, and concluded that the drug was too toxic for use in leprosy. In 1956, Leiker ${ }^{10}$ described 3 cases of the 'mononucleosis syndrome' with 1 death; he concluded that it was not glandular fever but was likely to be due to a 
delayed allergic reaction. After this paper there was a lapse of 25 years with no further reports until 2 papers appeared in $1981^{11,12}$ describing a reappearance of the sulphone syndrome, confirming the clinical picture described by the early writers, but showing that the leucocytosis is not always a mononucleosis; for example, 1 study ${ }^{11}$ describes a white cell count of $25,000 / \mathrm{cu} \mathrm{mm}$ with $59 \%$ neutrophils, $22 \%$ lymphocytes, $10 \%$ eosinophils and $9 \%$ mononuclears, while another ${ }^{12}$ describes a fatal case in which the number of leucocytes rose to $72,800 / \mathrm{cu}$ mm with $28 \%$ eosinophils. The fact that the patient who was the subject of the report ${ }^{11}$ was receiving only $50 \mathrm{mg} /$ day of dapsone emphasizes the fact that the sulphone syndrome is not dose-related.

The patients reported here developed hypersensitivity to dapsone and the G6PD activity was normal. All 3 of them-young and old - had fever, the onset was sudden, occurring $48 \mathrm{~h}$, as in Case 2, to 4 weeks, as in Case 1, after the initiation of dapsone therapy and had tender cervical lymph node enlargement. Cases 1 and 2 had generalized erythematous rash which was to a great extent macular, resolved on withdrawal of dapsone without resulting in exfoliative dermatitis and preceded the constitutional symptoms. A generalized rash was not observed in Case 3. In addition, Case 1 had developed methaemoglobinaemia and Case 2 had clinical and biochemical evidence of hepatitis. In Case 1 the lepra reaction which occurs usually within 6-12 months of therapy may not be a possibility. The drug was readministered in a smaller dose for confirmation in Case 1 and to observe the dose-related effect in Case 3 . The severity of the reaction in these patients was moderate and all attained resolution without the use of prednisolone, unlike those cases reported previously. ${ }^{11,}{ }^{12}$ Usually the symptoms of dapsone hypersensitivity reaction develop during the first 3-6 weeks after the start of therapy. But Case 2 developed symptoms 2 days after starting dapsone. This raises the question of whether the patient had taken dapsone in the past or developed cross-allergy to previous intake of any other sulphonamide or related drugs. Although the patient was unable to furnish details in this regard, such a justifiable possibility could not be ruled out. Case 3 had another interesting feature-a good response to another sulphonamide, sulphapyridine.

\section{Acknowledgments}

I wish to thank the Director-General of Health, Malaysia for permitting me to publish this article, and Mrs Noordin Hussin and Mr Selamat Omar for secretarial assistance.

\section{References}

${ }^{1}$ Alexander JO. Dapsone in the treatment of dermatitis herpetiformis. Lancet, 1955; 1: 1201-2. 
${ }^{2}$ Mohamed KN. Hailey-Hailey disease — case report. Med J Malaysia, 1983; 38: 188-90.

3 Barranco VP. Dapsone-other indications. Int J Dermatol, 1982; 21: 513-14.

${ }^{4}$ Stendhalo, Molin I, Dahlgren C. The inhibition of polymorphonuclear leucocyte cytotoxicity by dapsone. J Clin Invest, 1978; 62: 214-20.

${ }^{5}$ Graham WR. Adverse effects of dapsone. Int J Dermatol, 1975; 14: 494-500.

${ }^{6}$ Belmont A. Dapsone induced nephrotic syndrome. JAMA, 1967; 200: 262-3.

7 Young S, Mark JM. Dapsone and severe hypoalbuminaemia. Lancet, 1979; ii: 908.

${ }^{8}$ Allday EJ, Barnes J. Toxic effects of diaminodiphenyl—sulphone in the treatment of leprosy. Lancet, 1951; ii: 205-6.

${ }^{9}$ Lowe J, Smith M. Chemotherapy of leprosy in Nigeria. Int J Lepr, 1949; 17: 181-95.

${ }^{10}$ Leiker DJ. The mononucleosis syndrome in leprosy patients treated with sulfone. Int $J$ Lepr, 1956; 24: 402-5.

11 Tomeckik J, Catalano CJ. Dapsone hypersensitivity. Arch Dermatol, 1981; 117: 38-9.

12 Frey HM, Gershin AA, Borkowsky W, Bullock WE. Fatal reaction to dapsone during treatment of leprosy. Ann Intern Med, 1981; 94: 777-9. 